\title{
Asymptotic Freedom and QCD-a Historical Perspective
}

\author{
David J. Gross ${ }^{\mathrm{a}}$
}

${ }^{a}$ Kavli Institute for Theoretical Physics, University of California, Santa Barbara CA 93106-4030, USA

I describe the theoretical scene in the 1960's and the developments that led to the discovery of asymptotic freedom and to QCD.

\section{INTRODUCTION}

It was a pleasure to attend Loops and Legs in Quantum Field Theory, 2004, and to deliver a historical account of the origins of QCD. The talks delivered at this exciting meeting are a dramatic illustration of how far QCD has developed since its inception thirty years ago. Current and forthcoming experiments are performing tests of QCD with amazing precision, and theoretical calculations of perturbative QCD are truly heroic. In particular it was especially satisfying for me to meet at this conference some of the people, who over the last 30 years have calculated the two, three and four loop corrections to the $\beta$-function that we calculated to one loop order 31 years ago.

\section{THE THEORETICAL SCENE}

I would like first to describe the scene in theoretical particle physics, as I saw it in the early 1960's at Berkeley, when I started as a graduate student. The state of particle physics was then almost the complete opposite of today. It was a period of experimental supremacy and theoretical impotence. The construction and utilization of major accelerators were proceeding at full steam. Experimental discoveries and surprises appeared every few months. There was hardly any theory to speak of. The emphasis was on phenomenology, and there were only small islands of theoretical advances here and there. Field theory was in disgrace; S-Matrix theory was in full bloom. Symmetries were all the rage. The field was divided into the study of the weak and the strong interactions. In the case of the weak interactions, there was a rather successful phenomenological theory, but not much new data. The strong interactions were where the experimental and theoretical action was, particularly at Berkeley. They were regarded as especially unfathomable. The prevalent feeling was that it would take a very long time to understand the strong interactions and that it would require revolutionary concepts. For a young graduate student this was clearly the major challenge. The feeling at the time was well expressed by Lev Landau in his last paper, called "Fundamental Problems," which appeared in a memorial volume to Wolfgang Pauli in 1959 [1] . In this paper he argued that quantum field theory had been nullified by the discovery of the zero charge problem. He said:

"It is well known that theoretical physics is at present almost helpless in dealing with the problem of strong interactions.... By now the nullification of the theory is tacitly accepted even by theoretical physicists who profess to dispute it. This is evident from the almost complete disappearance of papers on meson theory and particularly from Dyson's assertion that the correct theory will not be found in the next hundred years."

Let us explore the theoretical milieu at this time.

\subsection{Quantum field theory}

Quantum field theory was originally developed for the treatment of electrodynamics almost immediately after the completion of quantum mechanics and the discovery of the Dirac equation. 
It seemed to be the natural tool for describing the dynamics of elementary particles. The application of quantum field theory had important early success. Fermi formulated a powerful and accurate phenomenological theory of beta decay, which was to serve as a framework for exploring the weak interactions for three decades. Yukawa proposed a field theory to describe the nuclear force and predicted the existence of heavy mesons, which were soon discovered. On the other hand, the theory was confronted from the beginning with severe difficulties. These included the infinities that appeared as soon as one went beyond lowest order perturbation theory, as well as the lack of any non-perturbative understanding of dynamics. By the 1950's the suspicion of field theory had deepened to the point that a powerful dogma emerged-that field theory was fundamentally wrong, especially in its application to the strong interactions. The renormalization procedure, developed by Richard Feynman, Julian Schwinger, Sin-itiro Tomanaga and Freeman Dyson, was spectacularly successful in Quantum Electrodynamics. However, the physical meaning of renormalization was not truly understood. The feeling of most was that renormalization was a trick. This was especially the case for the pioneering inventors of quantum field theory (for example Dirac and Wigner). They were prepared at the first drop of an infinity to renounce their belief in quantum field theory and to brace for the next revolution. However it was also the feeling of the younger leaders of the field, who had laid the foundations of perturbative quantum field theory and renormalization in the late '40's. The prevalent feeling was that renormalization simply swept the infinities under the rug, but that they were still there and rendered the notion of local fields meaningless. To quote Feynman, speaking at the 1961 Solvay conference [2], "I still hold to this belief and do not subscribe to the philosophy of renormalization." Field theory was almost totally perturbative at that time. The nonperturbative techniques that had been tried in the 1950's had all failed. The path integral, developed by Feynman in the late 1940's, which later proved so valuable for a nonperturbative formulation of quantum field theory as well as a tool for semiclassical expansions and numerical approximations, was almost completely forgotten. In a sense the Feynman rules were too successful. They were an immensely useful, picturesque and intuitive way of performing perturbation theory. However these alluring qualities also convinced many that all that was needed from field theory were these rules. They diverted attention from the non-perturbative dynamical issues facing field theory. In my first course on quantum field theory at Berkeley in 1965, I was taught that Field Theory $=$ Feynman Rules. In the United States, the main reason for the abandonment of field theory was simply that one could not calculate. American physicists are inveterate pragmatists. Quantum field theory had not proved to be a useful tool with which to make contact with the explosion of experimental discoveries. The early attempts in the 1950's to construct field theories of the strong interactions were total failures. In hindsight this was not surprising since a field theory of the strong interactions faced two enormous problems. First, which fields to use? Following Yukawa, the first attempts employed pion and nucleon fields. Soon, with the rapid proliferation of particles, it became evident that nothing was special about the nucleon or the pion. All the hadrons, the strange baryons and mesons as well as the higher spin recurrences of these, appeared to be equally fundamental. The obvious conclusion that all hadrons were composites of more fundamental constituents was thwarted by the fact thatno matter how hard one smashed hadrons at each one had not been able to liberate these hypothetical constituents. This was not analogous to the paradigm of atoms made of nucleons and electrons or of nuclei composed of nucleons. The idea of permanently bound, confined, constituents was unimaginable at the time. Second, since the pion-nucleon coupling was so large, perturbative expansions were useless. All attempts at non-perturbative analysis were unsuccessful. In the case of the weak interactions, the situation was somewhat better. Here one had an adequate effective theory-the four fermion Fermi interaction, which could be usefully employed, using perturbation theory to lowest order, to organize and understand the emerging experi- 
mental picture of the weak interactions. The fact that this theory was non-renormalizable meant that beyond the Born approximation it lost all predictive value. This disease increased the suspicion of field theory. Yang-Mills theory, which had appeared in the mid 1950's was not taken seriously. Attempts to apply Yang-Mills theory to the strong interactions focused on elevating global flavor symmetries to local gauge symmetries. This was problematic since these symmetries were not exact. In addition non-Abelian gauge theories apparently required massless vector mesons-clearly not a feature of the strong interactions. In the Soviet Union field theory was under even heavier attack, for somewhat different reasons. Landau and collaborators, in the late 1950 's, studied the high energy behavior of quantum electrodynamics. They explored the relation between the physical electric charge and the bare electric charge (essentially the electric charge that controls the physics at energies of order the ultraviolet cutoff). They concluded, on the basis of their approximations, that the physical charge vanishes, for any value of the bare charge as we let the ultraviolet cutoff become infinite (this is of course necessary to achieve a Lorentz invariant theory) [3]. "We reach the conclusion that within the limits of formal electrodynamics a point interaction is equivalent, for any intensity whatever, to no interaction at all." This is the famous problem of zero charge, a startling result that implied for Landau that "weak coupling electrodynamics is a theory, which is, fundamentally, logically incomplete." [4] . This problem occurs in any non-asymptotically-free theory. Even today, many of us believe that a non-asymptotically-free theory such as QED, if taken by itself, is inconsistent at very high energies. In the case of QED this is only an academic problem, since the trouble shows up only at enormously high energy. However in the case of the strong interactions, it was an immediate catastrophe. In the Soviet Union this was thought to be a compelling reason why field theory was wrong. Landau decreed that [1] "We are driven to the conclusion that the Hamiltonian method for strong interaction is dead and must be buried, although of course with deserved honor." Under the influence of Landau and Pomeranchuk, a generation of physicists was forbidden to work on field theory. One might wonder why the discovery of the zero charge problem did not inspire a search for asymptotically free theories that would be free of this disease. The answer, I think, is twofold. First, many other theories were explored-in each case they behaved as QED. Second, Landau and Pomeranchuk concluded, I think, that this problem was inherent in any quantum field theory, that an asymptotically free theory could not exist.

\subsection{The bootstrap}

The bootstrap theory rested on two principles, both more philosophical than scientific. First, local fields were not directly measurable. Thus they were unphysical and meaningless. Instead, one should formulate the theory using only observables. The basic observables are the S-Matrix elements measured in scattering experiments. Microscopic dynamics was renounced. Field theory was to be replaced by S-matrix theory; a theory based on general principles, such as unitarity and analyticity, but with no fundamental microscopic Hamiltonian. The basic dynamical idea was that there was a unique S-Matrix that obeyed these principles. It could be determined without the unphysical demand of fundamental constituents or equations of motion that was inherent in field theory In hindsight, it is clear that the bootstrap was born from the frustration of being unable to calculate anything using field theory. All models and approximations produced conflicts with some dearly held principle. If it was so difficult to construct an S-Matrix that was consistent with sacred principles then maybe these general principles had a unique manifestation. The second principle of the bootstrap was that there were no elementary particles. The way to deal with the increasing number of candidates for elementary status was to proclaim that all were equally fundamental, all were dynamical bound states of each other. This was called Nuclear Democracy, and was a response to the proliferation of candidates for fundamental building blocks. The bootstrap idea was immensely popular in the early 1960's, for a variety of reasons. Superseding quantum field theory, it rested on the solid principles of 
causality and unitarity. It was real and physical. It promised to be very predictive, indeed to provide a unique value for all observables. The bootstrap promised that this hope would be realized already in the theory of the strong interactions. This is of course false. We now know that there are an infinite number of consistent S-Matrices that satisfy all the sacred principles. One can take any non-Abelian gauge theory, with any gauge group, and many sets of fermions (as long as there are not too many to destroy asymptotic freedom.) The hope for uniqueness must wait for a higher level of unification. In Berkeley, as in the Soviet Union, S-Matrix theory was supreme, and a generation of young theorists was raised ignorant of field theory. Even on the calmer East Coast S-Matrix theory swept the field. For example, I quote Marvin Goldberger who said [7],

\begin{abstract}
"My own feeling is that we have learned a great deal from field theory... that I am quite happy to discard it as an old, but rather friendly, mistress who I would be willing to recognize on the street if I should encounter her again. From a philosophical point of view and certainly from a practical one the $S$-matrix approach at the moment seems to me by far the most attractive."
\end{abstract}

S-Matrix theory had some notable successes, the early application of dispersion relations and the development of Regge pole theory. However, there were drawbacks to a theory that was based on the principle that there was no theory, at least in the traditional sense. As Francis Low said [9],

"The distinction between S-Matrix theory and field theory is, on the one hand, between a set of equations that are not formulated, and on the other hand between a set of equations that are formulated if you knew what they were and for which you do not know whether there is a solution or not."

Nonetheless, until 1973 it was not thought proper to use field theory without apologies. For example as late as the NAL conference of 1972, Mur- ray Gell-Mann ended his talk on quarks with the summary [10],

"Let us end by emphasizing our main point, that it may well be possible to construct an explicit theory of hadrons, based on quarks and some kind of glue, treated as fictitious, but with enough physical properties abstracted and applied to real hadrons to constitute a complete theory. Since the entities we start with are fictitious, there is no need for any conflict with the bootstrap or conventional dual parton point of view."

\subsection{Symmetries}

If dynamics was impossible, one could at least explore the symmetries of the strong interactions. The biggest advance of the early 1960's was the discovery of an approximate symmetry of hadrons, $S U(3)$, by Gell-Mann and Yuval Neeman, and then the beginning of the understanding of spontaneously broken chiral symmetry. Since the relevant degrees of freedom, especially color, were totally hidden from view due to confinement, the emphasis was on flavor, which was directly observable. This emphasis was enhanced because of the success of $S U(3)$. Nowadays we realize that $S U(3)$ is an accidental symmetry, which arises simply because a few quarks (the up, down and strange quarks) are relatively light compared to the scale of the strong interactions. At the time it was regarded as a deep symmetry of the strong interactions, and many attempts were made to generalize it and use it as a springboard for a theory of hadrons. The most successful attempt was Gell-Mann's algebra of currents [12]. In an important and beautiful paper, he outlined a program for abstracting relations from a field theory, keeping the ones that might be generally true and then throwing the field theory away [12],

"In order to obtain such relations that we conjecture to be true, we use the method of abstraction from a Lagrangian field theory model. In other words, we construct a mathe- 
matical theory of the strongly interacting particles, which may or may not have anything to do with reality, find suitable algebraic relations that hold in the model, postulate their validity, and then throw away the model. We may compare this process to a method sometimes employed in French cuisine: a piece of pheasant meat is cooked between two slices of veal, which are then discarded."

This paper made quite an impression, especially on impoverished graduate students like me, who could only dream of eating such a meal. It was a marvelous approach. It gave one the freedom to play with the forbidden fruit of field theory, abstract what one wanted from it, all without having to believe in the theory. The only problem was that it was not clear what principle determined what to abstract? The other problem with this approach was that it diverted attention from dynamical issues. The most dramatic example of this is Gell-Mann and George Zweig's hypothesis of quarks [13], the most important consequence of the discovery of SU(3). The fact was that hadrons looked as if they were composed of (colored) quarks whose masses (either the current quark masses or the constituent quark masses) were quite small. Color had been introduced by Yoichiro Nambu [14] , M.Y. Han and Nambu [15] and O.W. Greenberg [16]. Nambu's motivation for color was two-fold, first to offer an explanation of why only (what we would now call) color singlet hadrons exist by postulating a strong force (but with no specification as to what kind of force) coupled to color which was responsible for the fact that color neutral states were lighter than colored states. The second motivation, explored with Han was the desire to construct models in which the quarks had integer valued electric charges. Greenberg's motivation was to explain the strange statistics of non-relativistic quark model hadronic bound states (a concern of Nambu's as well). He introduced parastatistics for this purpose, which equally well solved the statistics problem, but clouded the dynamical significance of this quantum number. Yet quarks had not been seen, even when energies wereachieved that were ten times the threshold for their production. This was not analogous to atoms made of nuclei and electrons or to nuclei made of nucleons. The non-relativistic quark model simply did not make sense. The conclusion was that quarks were fictitious, mathematical devices. With this attitude one could ignore the apparently insoluble dynamical problems that arose if one tried to imagine that quarks were real. This attitude towards quarks persisted until 1973 and beyond. Quarks clearly did not exist as real particles, therefore they were fictitious devices (see Gell-Mann above). One might "abstract" properties of quarks from some model, but one was not allowed to believe in their reality or to take the models too seriously.

\subsection{Experiment}

This was a period of great experimental excitement. However, I would like to discuss an interesting phenomenon, in which theorists and experimentalists reinforced each other's conviction that the secret of the strong interactions lay in the high-energy behavior of scattering amplitudes at low momentum transfer. Early scattering experiments concentrated, for obvious reasons, on the events that had the largest rates. In the case of the strong interactions, this meant searching for resonant bumps or probing near forward scattering, where the cross section was largest. It was not at all realized by theorists that the secret of hadronic dynamics could be revealed by experiments at large momentum transfer that probed the short distance structure of hadrons. Instead, prompted by the regularities that were discovered at low momentum transfer, theorists developed an explanation based on the theory of Regge poles. This was the only strong interaction dynamics that was understood, for which there was a real theory. Therefore theorists concluded that Regge behavior must be very important and forward scattering experiments were deemed to be the major tool of discovery. Regge theory was soon incorporated into the bootstrap program as a boundary condition. In response to this theoretical enthusiasm, the interest of experimentalists in forward scattering was enhanced. Opportuni- 
ties to probe the less easily accessible domains of large momentum transfer were ignored. Only much later, after the impact of the deep-inelastic scattering experiments that had been ridiculed by many as unpromising, was it understood that the most informative experiments were those at large momentum transfers that probe short or lightlike distances. It used to be the case that when a new accelerator was initiated one of the first and most important experiments to be performed was the measurement of the total p-p cross section. Nowadays, this experiment is regarded with little interest, even though the explanation of Regge behavior remains an interesting, unsolved and complicated problem for QCD. Ironically, one of the principal justifications for this experiment today is simply to calibrate the luminosity of the machine.

\section{MY ROAD TO ASYMPTOTIC FREE- DOM}

\subsection{From N/D to QCD}

I was a graduate student at Berkeley at the height of the bootstrap and S-Matrix theory. My $\mathrm{Ph} . \mathrm{D}$. thesis was written under the supervision of Geoff Chew, the main guru of the bootstrap, on multi-body $N / D$ equations. I can remember the precise moment at which I was disillusioned with the bootstrap program. This was at the 1966 Rochester meeting, held at Berkeley. Francis Low, in the session following his talk, remarked that the bootstrap was less of a theory than a tautology [8],

"I believe that when you find that the particles that are there in S-Matrix theory, with crossing matrices and all the formalism, satisfy all these conditions, all you are doing is showing that the $S$ matrix is consistent with the world the way it is; that is the particles have put themselves there in such a way that it works out, but you have not necessarily explained that they are there."

For example, the then popular finite energy sum rules (whereby one derived relations for measur- able quantities by saturating dispersion relations with a finite number of resonance poles on the one hand and relating these to the assumed Regge asymptotic behavior on the other) were not so much predictive equations, but merely checks of axioms (analyticity, unitarity) using models and fits of experimental data. I was very impressed with this remark and longed to find a more powerful dynamical scheme. This was the heyday of current algebra, and the air was buzzing with marvelous results. I was very impressed by the fact that one could assume a certain structure of current commutators and derive measurable results. The most dramatic of these was the AdlerWeisberger relation that had just appeared [18]. Clearly the properties of these currents placed strong restrictions on hadronic dynamics. The most popular scheme then was current algebra. Gell-Mann and Roger Dashen were trying to use the commutators of certain components of the currents as a basis for strong interaction dynamics. After a while I concluded that this approach was also tautological-all it did was test the validity of the symmetries of the strong interactions. This was apparent for vector $S U(3)$. However it was also true of chiral $S U(3)$, especially as the current algebra sum rules were interpreted, by Weinberg and others, as low energy theorems for Goldstone bosons. This scheme could not be a basis for a complete dynamical theory. I studied the less understood properties of the algebra of local current densities. These were model dependent; but that was fine, they therefore might contain dynamical information that went beyond statements of global symmetry. Furthermore, as was soon realized, one could check ones' assumptions about the structure of local current algebra by deriving sum rules that could be tested in deep-inelastic lepton-hadron scattering experiments. James Bjorken's 1967 paper [20,21]), on the application of $U(6) \times U(6)$, particularly influenced me. In the spring of 1968 Curtis Callan and I proposed a sum rule to test the then popular "Sugawara model," a dynamical model of local currents, in which the energy momentum tensor was expressed as a product of currents [22]. The hope was that the algebraic properties of the currents and the expression for the 
Hamiltonian in terms of these would be enough to have a complete theory. (This idea actually works in the now very popular two-dimensional conformal field theories). Our goal was slightly more modest-to test the hypothesis by exploiting the fact that in this theory the operator product expansion of the currents contained the energy momentum tensor with a known coefficient. Thus we could derive a sum rule for the structure functions [23] that could be measured in deepinelastic electron-proton scattering. In the fall of 1968, Bjorken noted that this sum rule, as well as dimensional arguments, would suggest the scaling of deep-inelastic scattering cross sections [24]. This prediction was shortly confirmed by the new experiments at SLAC, which were to play such an important role in elucidating the structure of hadrons [25]. Shortly thereafter Callan and I discovered that by measuring the ratio, $R=\frac{\sigma_{L}}{\sigma_{T}}$, (where $\sigma_{L}\left(\sigma_{T}\right)$ is the cross section for the scattering of longitudinal or transverse polarized virtual photons), one could determine the spin of the charged constituents of the nucleon [26]. We evaluated the moments of the deep-inelastic structure functions in terms of the equal time commutators of the electromagnetic using specific models for these-the algebra of fields in which the current was proportional to a spin-one field on the one hand, and the quark-gluon model on the other. In this popular model quarks interacted through an Abelian gauge field (which could, of course, be massive) coupled to baryon number. The gauge dynamics of the gluon had never been explored, and I do not think that the model had been used to calculate anything until then. We discovered that $R$ depended crucially on the spin of the constituents. If the constituents had spin zero or one, then $\sigma_{T}=0$, but if they had spin- $\frac{1}{2}$, then $\sigma_{L}=0$. This was a rather dramatic result. The experiments quickly showed that $\sigma_{L}$ was very small. These SLAC deep-inelastic scattering experiments had a profound impact on me. They clearly showed that the proton behaved, when observed over short times, as if it was made out of point-like objects of spin one-half. In the spring of 1969, which I spent at CERN, Chris Llewelynn-Smith and I analyzed the sum rules that followed for deep-inelastic neutrino-nucleon scattering using similar methods [27]. We were clearly motivated by the experiments that were then being performed at CERN. We derived a sum rule that measured the baryon number of the charged constituents of the proton. The experiments soon indicated that the constituents of the proton had baryon number $\frac{1}{3}$-in other words again they looked like quarks. I was then totally convinced of the reality of quarks. They had to be more than just mnemonic devices for summarizing hadronic symmetries, as they were then universally regarded. They had to be physical point-like constituents of the nucleon. But how could that be? Surely strong interactions must exist between the quarks that would smear out their point-like behavior. After the experiments at SLAC, Feynman came up with his parton picture of deep-inelastic scattering. This was a very picturesque and intuitive way of describing deepinelastic scattering in terms of assumed pointlike constituents-partons [28]. It complemented the approach to deep-inelastic scattering based on the operator product of currents, and had the advantage of being extendible to other processes [29]. The parton model allowed one to make predictions with ease, ignoring the dynamical issues at hand. I felt more comfortable with the approach based on assuming properties of current products at short distances. I felt somewhat uneasy about the extensions of the parton model to processes that were not truly dominated by short distance singularities. At CERN I studied, with Julius Wess, the consequences of exact scale and conformal invariance [30]. However, I soon realized that in a field theoretic context only a free, non-interacting theory could produce exact scaling. This became very clear to me in 1970, when I came to Princeton, where my colleague Curtis Callan (and Kurt Symanzik) had rediscovered the renormalization group equations [33], [34], which they presented as a consequence of a scale invariance anomaly [36]. Their work made it abundantly clear that once one introduced interactions into the theory, scaling, as well as my beloved sum rules, went down the tube. Yet the experiments indicated that scaling was in fine shape. But one could hardly turn off the interactions between the quarks, or make them very weak, since 
then one would expect hadrons to break up easily into their quark constituents. Why then had no one ever observed free quarks? This paradox and the search for an explanation of scaling were to preoccupy me for the following four years.

\subsection{How to explain scaling}

About the same time that all this was happening, string theory was discovered, in one of the most bizarre turn of events in the history of physics. In 1968 Gabrielle Veneziano came up with a remarkably simple formula that summarized many features of hadronic scattering. It had Regge asymptotic behavior in one channel and narrow resonance saturation in the other [31]. This formula was soon generalized to multiparticle S-Matrix amplitudes and attracted much attention. The dual resonance model was born, the last serious attempt to implement the bootstrap. It was only truly understood as a theory of quantized strings in 1972. I worked on this theory for two years, first at CERN and then at Princeton with John Schwarz and Andre Neveu. At first I felt that this model, which captured many of the features of hadronic scattering, might provide the long sought alternative to a field theory of the strong interactions. However by 1971 I realized that there was no way that this model could explain scaling, and I felt strongly that scaling was the paramount feature of the strong interactions. In fact the dual resonance model lead to incredibly soft behavior at large momentum transfer, quite the opposite of the hard scaling observed. Furthermore, it was clear that it required for consistency many features that were totally unrealistic for the strong interactions-massless vector and tensor particles. These features later became the motivation for the hope that string theory may provide a comprehensive and unified theory of all the forces of nature. This hope remains strong. However the relevant energy scale is not $1 \mathrm{GeV}$ but rather $10^{19} \mathrm{GeV}$ ! The view that the scaling observed at SLAC was not a truly asymptotic phenomenon was rather widespread. The fact that scaling set in at rather low momentum transfers, "precocious scaling," reinforced this view. Thus the cognoscenti of the renormalization group (Wilson, Polyakov, and others) be- lieved that the non-canonical scaling indicative of a non-trivial fixed point of the renormalization group would appear at higher energies. Much happened during the next two years. Gerhard 't Hooft's spectacular work [32] on the renormalizability of Yang-Mills theory, reintroduced nonAbelian gauge theories to the community. The electroweak theory of Sheldon Glashow, Weinberg and Abdus Salam was revived. Field theory became popular again, at least in application to the weak interactions. The path integral reemerged from obscurity. Kenneth Wilson's development of the operator product expansion provided a tool that could be applied to the analysis of deep-inelastic scattering. Most important from my point of view was the revival of the renormalization group by Wilson [37]. The renormalization group stems from the fundamental work of Gell-Mann and Low [33], E. Stueckelberg and A. Petermann [34] and Bogoliubov and Shirkov [35] - This work was neglected for many years, partly because it seemed to provide only information about physics for large space-like momenta, which are of no direct physical interest. Also, before the discovery of asymptotic freedom, the ultraviolet behavior was not calculable using perturbative methods, and there were no others. Thus it appeared that the renormalization group provided a framework in which one could discuss, but not calculate, the asymptotic behavior of amplitudes in a physically uninteresting region. Wilson's development of the operator product expansion provided a new tool that could be applied to the analysis of deep-inelastic scattering. The CallanSymanzik equations simplified the renormalization group analysis, which was then applied to the Wilson expansion $[39,42]$. The operator product analysis was extended to the light cone, the relevant region for deep-inelastic scattering [38] . Most influential was Wilson's deep understanding of renormalization, which he was then applying to critical behavior. Wilson gave a series of lectures at Princeton in the spring of 1972 [40]. These had a great impact on many of the participants, certainly on me. 


\subsection{The plan}

By the end of 1972, I had learned enough field theory, especially renormalization group methods from Ken Wilson, to tackle the problem of scaling head on. I decided, quite deliberately, to prove that local field theory could not explain the experimental fact of scaling and thus was not an appropriate framework for the description of the strong interactions. Thus, deep-inelastic scattering would finally settle the issue as to the validity of quantum field theory. The plan of the attack was twofold. First, I would prove that "ultraviolet stability," the vanishing of the effective coupling at short distances, later called asymptotic freedom, was necessary to explain scaling. Second, I would show that there existed no asymptotically free field theories. The latter was to be expected. After all the paradigm of quantum field theory-Quantum Electrodynamics (QED)was infrared stable; in other words, the effective charge grew larger at short distances and no one had ever constructed a theory in which the opposite occurred. Charge renormalization is nothing more (certainly in the case of QED) than vacuum polarization. The vacuum or the ground state of a relativistic quantum mechanical system can be thought of as a medium of virtual particles. In QED the vacuum contains virtual electron-positron pairs. If a charge, $e_{0}$, is put in this medium, it polarizes it. Such a medium with virtual electric dipoles will screen the charge and the actual, observable, charge e, will differ from $e_{0}$ as $\frac{e_{0}}{\epsilon}$, where $\epsilon$ is the dielectric constant. Now $\epsilon$ is frequency dependent (or energy or distance dependent). To deal with this one can introduce the notion of an effective coupling $e(r)$, which governs the force at a distance $r$. As $r$ increases, there is more medium that screens, thus $e(r)$ decreases with increasing $r$, and correspondingly increases with decreasing $r$. The $\beta$-function, which is simply minus the derivative of $\log [e(r)]$ with respect to $\log (r)$, is therefore positive. If the effective coupling were, contrary to QED, to decrease at short distances, one might explain how the strong interactions turn off in this regime and produce scaling. Indeed, one might suspect that this is the only way to get point-like behavior at short distances. It was well understood, due to Wilson's work and its application to deep-inelastic scattering, that one might expect to get scaling in a quantum field theory at a fixed point of the renormalization group. However this scaling would not have canonical, free-field-theory-like behavior. Such behavior would mean that the scaling dimensions of the operators that appear in the product of electromagnetic currents at lightlike distances had canonical, free field dimensions. This seemed unlikely. I knew that if the fields themselves had canonical dimensions, then for many theories this implied that the theory was trivial, i.e., free. Surely this was also true if the composite operators that dominated the amplitudes for deep-inelastic scattering had canonical dimensions. By the spring of 1973, Callan and I had completed a proof of this argument, extending an idea of Giorgio Parisi [41] to all renormalizable field theories, with the exception of nonAbelian gauge theories. The essential idea was to prove that the vanishing anomalous dimensions of the composite operators, at an assumed fixed point of the renormalization group, implied the vanishing anomalous dimensions of the fields. This then implied that the theory was free at this fixed point. The conclusion was that naive scaling could be explained only if the assumed fixed point of the renormalization group was at the origin of coupling space- i.e., the theory must be asymptotically free [42]. Non-Abelian gauge theories were not included in the argument since both arguments broke down for these theories. The discovery of asymptotic freedom made this omission irrelevant. The second part of the argument was to show that there were no asymptotically free theories at all. I had set up the formalism to analyze the most general renormalizable field theory of fermions and scalars- again excluding non-Abelian gauge theories. This was not difficult, since to investigate asymptotic freedom it suffices to study the behavior of the $\beta$ functions in the vicinity of the origin of coupling constant space, i.e., in lowest order perturbation theory (one-loop approximation). I almost had a complete proof but was stuck on my inability to prove a necessary inequality. I discussed the issue with Sidney Coleman, who was spending the spring semester in Princeton. He came up with 
the missing ingredient, and added some other crucial points -and we had a proof that no renormalizable field theory that consisted of theories with arbitrary Yukawa, scalar or Abelian gauge interactions could be asymptotically free [43]. Tony Zee had also been studying this. He too was well aware of the advantages of an asymptotically free theory and was searching for one. He derived, at the same time, a partial result, indicating the lack of asymptotic freedom in theories with $S U(N)$ invariant Yukawa couplings [44].

\subsection{The discovery of asymptotic freedom}

Frank Wilczek started work with me in the fall of 1972. He had come to Princeton as a mathematics student, but soon discovered that he was really interested in particle physics. He switched to the physics department, after taking my field theory course in 1971, and started to work with me. My way of dealing with students, then and now, was to involve them closely with my current work and very often to work with them directly. This was certainly the case with Frank, who functioned more as a collaborator than a student from the beginning. I told him about my program to determine whether quantum field theory could account for scaling. We decided that we would calculate the $\beta$-function for Yang-Mills theory. This was the one hole in the line of argument I was pursuing. It had not been filled largely because Yang-Mills theory still seemed strange and difficult. Few calculations beyond the Born approximation had ever been done. Frank was interested in this calculation for other reasons as well. Yang-Mills theory was already in use for the electro-weak interactions, and he was interested in understanding how these behaved at high energy. Coleman, who was visiting in Princeton, asked me at one point whether anyone had ever calculated the $\beta$-function for Yang-Mills theory. I told him that we were working on this. He expressed interest because he had asked his student, H. David Politzer, to generalize the mechanism he had explored with Eric Weinberg-that of dynamical symmetry breaking of an Abelian gauge theory- to the non-Abelian case. An important ingredient was the knowledge of the renormalization flow, to decide whether lowest order pertur- bation theory could be a reliable guide to the behavior of the energy functional. Indeed, Politzer went ahead with his own calculation of the $\beta$ function for Yang-Mills theory. Our calculation proceeded slowly. I was involved in the other parts of my program and there were some tough issues to resolve. We first tried to prove on general grounds, using spectral representations and unitarity, that the theory could not be asymptotically free, generalizing the arguments of Coleman and me to this case. This did not work, so we proceeded to calculate the $\beta$-function for a YangMills theory. Today this calculation is regarded as quite simple and even assigned as a homework problem in quantum field theory courses. At the time it was not so easy. This change in attitude is the analogue, in theoretical physics, of the familiar phenomenon in experimental physics whereby yesterday's great discovery becomes today's background. It is always easier to do a calculation when you know what the result is and you are sure that the methods make sense. One problem we had to face was that of gauge invariance. Unlike QED, where the charge renormalization was trivially gauge invariant (because the photon is neutral), the renormalization constants in QCD were all gauge dependent. However the physics could not depend on the gauge. Another issue was the choice of regularization. Dimensional regularization had not really been developed yet, and we had to convince ourselves that the oneloop $\beta$-function was insensitive to the regularization used. We did the calculation in an arbitrary gauge. Since we knew that the answer had to be gauge invariant, we could use gauge invariance as a check on our arithmetic. This was good since we both kept on making mistakes. In February the pace picked up, and we completed the calculation in a spurt of activity. At one point a sign error in one term convinced us that the theory was, as expected, non-asymptotically free. As I sat down to put it all together and to write up our results, I caught the error. At almost the same time Politzer finished his calculation and we compared, through Sidney, our results. The agreement was satisfying. A month or two after this Symanzik passed through Princeton and told us that 't Hooft had made a remark in a question ses- 
sion during a meeting at Marseilles the previous fall to the effect that non-Abelian gauge theories worked in the same way as an asymptotically free scalar theory he had been playing with. ${ }^{1}$ 't Hooft did not publish ${ }^{2}$ and apparently did not realize the significance for scaling and meeting for the strong interactions. Why are non-Abelian gauge theories asymptotically free? Today we can understand this in a very physical fashion, although it was certainly not so clear in 1973 . It is instructive to interrupt the historical narrative and explain, in modern terms, why QCD is asymptotically free. The easiest way to understand this is by considering the magnetic screening properties of the vacuum [47]. In a relativistic theory one can calculate the dielectric constant, $\epsilon$, in terms of the magnetic permeability, $\mu$, since $\epsilon \mu=1$ (in units where $c=$ velocity of light $=1)$. In classical physics all media are diamagnetic. This is because, classically, all magnets arise from electric currents and the response of a system to an applied magnetic field is to set up currents that act to decrease the field (Lenz's law). Thus $\mu<1$, a situation that corresponds to electric screening or $\epsilon>1$. However, in quantum mechanical systems paramagnetism is possible. This is the case in non-Abelian gauge theories where the gluons are charged particles of spin one. They behave as permanent color magnetic dipoles that align themselves parallel to an applied external field increasing its magnitude and producing $\mu>1$. We can therefore regard the anti-screening of the Yang-Mills vacuum as paramagnetism. QCD is asymptotically free because the anti-screening of the gluons overcomes the screening due to the quarks. The arithmetic works as follows. The contribution to $\epsilon$ (in some units) from a particle of charge $q$ is $-\frac{q^{2}}{3}$, arising from ordinary dielectric (or diamagnetic) screening. If the particle has spin $s$ (and thus a permanent dipole moment $\gamma s)$, it contributes $(\gamma s)^{2}$ to $\mu$. Thus a spin one gluon (with $\gamma=2$, as in Yang-Mills theory) gives

\footnotetext{
${ }^{1}$ This scalar theory was ruled out, as Coleman and I argued [43], since one could prove it had no ground state and therefore was unstable.

${ }^{2}$ Symanziks paper, in the proceedings of the Marseilles meeting (1973), presents the issue of the ultraviolet behavior of Yang-Milss theory as an open question.
}

a contribution to $\mu$ of

$$
\delta \mu=\left(-1 / 3+2^{2}\right) q^{2}=\frac{11}{3} q^{2} ;
$$

whereas a spin one-half quark contributes,

$$
\delta \mu=-\left(-1 / 3+\left(2 \times \frac{1}{2}\right)^{2}\right) q^{2}=-\frac{2}{3} q^{2} .
$$

(the extra minus arises because quarks are fermions). In any case, the upshot is that as long as there are not too many quarks the antiscreening of the gluons wins out over the screening of the quarks. The formula for the $\beta$-function of a non-Abelian gauge theory is given by

$$
\begin{gathered}
\left.\beta(\alpha) \equiv \mu \frac{d}{d \mu} \alpha(\mu)\right|_{\alpha_{\text {barefixed }}} \\
=\frac{\alpha^{2}}{\pi} b_{1}+\left(\frac{\alpha^{2}}{\pi}\right)^{2} b_{2}+\ldots
\end{gathered}
$$

where

$$
\alpha=\frac{g^{2}}{4 \pi}
$$

Our result was that

$b_{1}=-\left[\frac{11}{6} C_{A}-\frac{2}{3} \sum_{R} n_{R} T_{R}\right]$

Here $C_{R}$ is the eigenvalue of the quadratic Casimir operator in the representation $R$ of $S U(N)$ (for the adjoint representation $C_{A}=N$, for the fundamental $\left.C_{F}=\frac{N^{2}-1}{N}\right), T_{R}$ is trace of the square of the generators for the representation $R$ of $S U(N)\left(T_{A}=\mathrm{N}\right.$ and $\left.T_{F}=\frac{1}{2}\right)$, and $n_{R}$ is the number of fermions in the representation R. In the case of a $S U(3)$ gauge group such as $\mathrm{QCD}, C_{A}=3, T_{F}=2$, and thus $b_{1}=-\left[\frac{11}{2}-\frac{n}{3}\right]$. Thus one can tolerate as many as 16 triplets of quarks before losing asymptotic freedom.

\section{NON-ABELIAN GAUGE THEORIES OF THE STRONG INTERACTIONS}

For me the discovery of asymptotic freedom was totally unexpected. Like an atheist who has just received a message from a burning bush, I became an immediate true believer. Field theory wasn't wrong-instead scaling must be explained by an asymptotically free gauge theory of the 
strong interactions. Our first paper contained, in addition to the report of the asymptotic freedom of Yang-Mills theory, the hypothesis that this could offer an explanation for scaling, a remark that there would be logarithmic violations of scaling and most important of all the suggestion that the strong interactions must be based on a color gauge theory. The first paragraph of our paper reads [45]:

Non-Abelian gauge theories have received much attention recently as a means of constructing unified and renormalizable theories of the weak and electromagnetic interactions. In this note we report on an investigation of the ultraviolet asymptotic behavior of such theories. We have found that they possess the remarkable feature, perhaps unique among renormalizable theories, of asymptotically approaching free-field theory. Such asymptotically free theories will exhibit, for matrix elements of currents between onmass-shell states, Bjorken scaling. We therefore suggest that one should look to a non-Abelian gauge theory of the strong interactions to provide the explanation for Bjorken scaling, which has so far eluded field theoretic understanding."

We had a specific theory in mind, namely what became known as QCD. Since the deep-inelastic experiments indicated that the charged constituents of the nucleon were quarks, the gluons had to be flavor neutral. Thus the gluons could not couple to flavor. We were very aware of the growing arguments for the color quantum number. Not just the quark model spectroscopy that was the original motivation of Han and Nambu and Greenberg [15], [16], but the counting factor (of three) that went into the evaluation of the $\pi^{0} \rightarrow 2 \gamma$ decay rate from the axial anomaly ${ }^{3}$, and the factor of three that color provided in the total $e^{+}-e^{-}$annihilation cross section. Thus the glu-

\footnotetext{
${ }^{3}$ This had been recently emphasized by William Bardeen, Harald Fritzsch and Gell-Mann [48].
}

ons could couple to color and all would be well. Thus we proposed [45]:

"One particularly appealing model is
based on three triplets of fermions,
with Gell-Mann's $S U(3) \times S U(3)$ as
a global symmetry and a SU $(3)$
'color' gauge group to provide the
strong interactions. That is, the
generators of the strong interaction
gauge group commute with ordinary
SU(3) $\times$ SU(3) currents and mix
quarks with the same isospin and hy-
percharge but different 'color'. In
such a model the vector mesons are
(flavor) neutral, and the structure
of the operator product expansion
of electromagnetic or weak currents
is essentially that of the free quark
model (up to calculable logarithmic
corrections)."

The appearance of logarithmic corrections to scaling in asymptotically free theories had already been discussed by Callan and me, in our work on the need for an asymptotically free theory to obtain Bjorken scaling. We also analyzed deepinelastic scattering in an asymptotically free theory and discovered [42]

\section{"That in such asymptotically free the- ories naive scaling is violated by cal- culable logarithmic terms."}

Thus we were well aware what the form of the scaling deviations would be in such a theory. Wilczek and I had immediately started to calculate the logarithmic deviations from scaling. We were tremendously excited by the possibility of deriving exact experimental predictions from first principles that could conclusively test our asymptotically free theories of the strong interactions. We had already evaluated the asymptotic form of the flavor non-singlet structure functions, which were the easiest to calculate, at the time our Physical Review Letter was written, but did not have room to include the results. We immediately started to write a longer paper in which the structure of the theory would be spelled out in more detail and the dynamical issues would be 
addressed, especially the issue of confinement. In our letter we were rather noncommittal on this issue. We had tentatively concluded that Higgs mesons would destroy asymptotic freedom, but had only begun to explore the dynamical consequences of unbroken color symmetry. The only thing we were sure of was that [45]

"...perturbation theory is not trustworthy with respect to the stability of the symmetric theory nor to its particle content."

Politizer's paper appeared with ours [46]. He pointed out the asymptotic freedom of Yang-Mills theory and speculated on its implications for the dynamical symmetry breaking of these theories. In our second paper, written a few months later, we outlined in much greater detail the structure of asymptotically free gauge theories of the strong interactions and the predictions for the scaling violations in deep-inelastic scattering [55]. Actually the paper was delayed for about two months because we had problems with the singlet structure functions-due to the operator mixing of physical operators with ghost operators. This problem was similar to the issue of gauge invariance that had plagued us before. Here the problem was more severe. Physical operators, whose matrix elements were measurable in deep-inelastic scattering experiments, mixed under renormalization with ghost operators that could have no physical meaning. Finally we deferred the analysis of the singlet structure functions to a third paper [56], in which we resolved this issue. We showed that, even though this mixing was real and unavoidable, the ghost operators decoupled from physical measurements. In the second paper we discussed in detail the choice between symmetry breaking and unbroken symmetry and noted that [55]

"Another possibility is that the gauge symmetry is exact. At first, sight this would appear to be ridiculous since it would imply the existence of massless, strongly coupled vector mesons. However, in asymptotically free theories these naive expectations might be wrong. There may be little connection between the 'free' Lagrangian and the spectrum of states.... The infrared behavior of Green's functions in this case is determined by the strong-coupling limit of the theory. It may be very well that this infrared behavior is such so as to suppress all but color singlet states, and that the colored gauge fields as well as the quarks could be 'seen' in the large-Euclidean momentum region but never produced as real asymptotic states."

Steve Weinberg reacted immediately to asymptotic freedom. He wrote a paper in which he pointed out that in an asymptotically free gauge theory of the strong interactions the nonconservation of parity and strangeness can be calculated ignoring the strong interactions, and thus is of order $\alpha$, as observed. He also suggested that a theory with unbroken color symmetry could explain why we do not see quarks. There is a important difference between our respective conjectures. Weinberg argued that perhaps the infrared divergences, caused by the masslessness of the gluons in an unbroken color gauge theory, would make the rate of production of non-singlet states vanish. ${ }^{4}$ We argued that perhaps the growth of the effective coupling at large distances, the infrared behavior of the coupling caused by the flip side of asymptotic freedom ${ }^{5}$, would confine the quarks and gluons in color singlet states. In October 1973 Fritzsch, Gell-Mann and H. Leutwyler submitted a paper in which they discussed the "advantages of color octet gluon picture" [50]. Here they discussed the advantages of

\section{"abstracting properties of hadrons and their currents from a Yang-Mills gauge model based on colored quarks and color octet gluons."}

They discussed various models and pointed out the advantages of each. The first point was al-

\footnotetext{
${ }^{4}$ Today we believe in the existence in non-confining, Coulomb phases, with unbroken color symmetry, for some supersymmetric non-Abelian gauge theories.

${ }^{5}$ Later dubbed infrared slavery by Georgi and Glashow [76], a name invented by Sidney Coleman.
} 
ready discussed at the NAL high-energy physics conference in August 1972. There Gell-Mann and Fritzsch had discussed their program of "abstracting results from the quark-gluon model." They discussed various models and asked, "Should we regard the gluons as well as being color nonsinglets." They noted that if one assumed that the gluons were color octets then "an annoying asymmetry between quarks and gluons is removed." In that talk no dynamical theory was proposed and in most of the paper they "shall treat the vector gluon, for convenience, as a color singlet." [10] In October 1973 Fritzsch, Gell-Mann and Leutwyler also noted that in the non-relativistic quark model with a Coulomb potential mediated by vector gluons the potential is attractive in color singlet channels, which might explain why these are light. This point had been made previously by Harry Lipkin [57]. They also noted the asymptotic freedom of such theories, but did not regard this as an argument for scaling since "we conjecture that there might be a modification at high energies that produces true scaling." Finally they noted that the axial U(1) anomaly in a non-Abelian gauge theory might explain the notorious $U(1)$ problem, although they could not explain how, since the anomaly itself could be written as a total divergence. ${ }^{6}$

\section{THE EMERGENCE AND ACCEP- TANCE OF QCD}

Although it was clear to me that the strong interactions must be described by non-Abelian gauge theories, there were many problems. The experimental situation was far from clear, and the issue of confinement remained open. However, within a small community of physicists the acceptance of the theory was very rapid. New ideas in physics sometimes take years to percolate into the collective consciousness. However in rare cases such as this there is a change of perception analogous to a phase transition. Before asymptotic freedom it seemed that we were still far from a dynamical theory of hadrons; afterwards it seemed

\footnotetext{
${ }^{6}$ It required the discovery of instantons [52] to find the explanation of the $U(1)$ problem $[53,54]$.
}

clear that $\mathrm{QCD}^{7}$ was such a theory. Asymptotic freedom explained scaling at short distances and offered a mechanism for confinement at large distance. Suddenly it was clear that a non-Abelian gauge theory was consistent with everything we knew about the strong interactions. It could encompass all the successful strong interaction phenomenology of the past decade. Since the gluons were flavor neutral, the global flavor symmetries of the strong interactions, $\mathrm{SU}(3) \times \mathrm{SU}(3)$, were immediate consequences of the theory, as long as the masses of the quarks were small enough. ${ }^{8}$ Even more alluring was the fact that one could calculate. Since perturbation theory was trustworthy at short distances many problems could be tackled. Some theorists were immediately convinced, among them Guido Altarelli, Tom Appelquist, Callan, Coleman, Mary K. Gaillard, R. Gatto, Georgi, Glashow, John Kogut, Ben Lee, Luciano Maiani, Migdal, Polyakov, Politzer, Lennie Susskind, S. Weinberg, Zee. At large distances however perturbation theory was useless. In fact, even today after thirty-one years of study we still lack reliable, analytic tools for treating this region of QCD. This remains one of the most important, and woefully neglected, areas of theoretical particle physics. However, at the time the most important thing was to convince oneself that the idea of confinement was not inconsistent. One of the first steps in that direction was provided by lattice gauge theory. I first heard of Wilson's lattice gauge theory when I gave a lecture at Cornell in the late spring of 1973 . Wilson had started to think of this approach soon after asymptotic freedom was discovered. The lattice formulation of gauge theory (independently proposed by Polyakov) had the enormous advantage, as Wilson pointed out in the fall of 1973, that the strong coupling limit was particularly simple and exhibited confinement [59]. Thus one had at least a crude approximation in which confine-

\footnotetext{
${ }^{7}$ The name $Q C D$ first appeared in a review by Bill Marciano and Heinz Pagels [58], where it was attributed to Gell-Mann. It was such an appropriate name that no one could complain.

${ }^{8} \mathrm{I}$ refer of course to the mass parameters of the quarks in the Lagrangian, not the physical masses that are effectively infinite due to confinement.
} 
ment was exact. It is a very crude approximation, since to arrive at the continuum theory from the lattice theory one must take the weak coupling limit. However one could imagine that the property of confinement was not lost as one went continuously from strong to weak lattice coupling, i.e., there was no phase transition. Moreover one could, as advocated by Wilson, study this possibility numerically using Monte Carlo methods to construct the lattice partition function. However, the first quantitative results of this program did not emerge till the work of Creutz [60] in 1981. The ambitious program of calculating the hadronic mass spectrum has still not attained its goal, and still awaits the next generation of computers. Personally I derived much solace in the coming year from two examples of soluble twodimensional field theories. One was the $(\bar{\Psi} \Psi)^{2}$ theory that Neveu and I analyzed and solved for large N [61]. This provided a soluble example of an asymptotically free theory that underwent dimensional transmutation, solving its infrared problems by generating a dynamical fermion mass through spontaneous symmetry breaking. This provided a model of an asymptotically free theory, with no built in mass parameters. We could solve this model and check that it was consistent and physical. The other soluble model was two dimensional QCD, analyzed by t'Hooft in the large $\mathrm{N}$ limit [62]. Two dimensional gauge theories trivially confine color. This was realized quite early and discussed for Abelian gauge theory-the Schwinger model- by Aharon Casher, Kogut and Susskind, as a model for confinement in the fall of 1973 [63]. However $Q C D_{2}$ is a much better example. It has a spectrum of confined quarks which in many ways resembles the four dimensional world. These examples gave many of us total confidence in the consistency of the concept of confinement. It clearly was possible to have a theory whose basic fields do not correspond to asymptotic states, to particles that one can observe directly in the laboratory. Applications of the theory also began to appear. Two calculations of the $\beta$-function to two loop order were performed $[64,65]$, with the result that, in the notation of $(3), b_{2}=-\left[\frac{17}{12} C_{A}^{2}-\frac{1}{2} C_{F} T_{F} n-\frac{5}{6} C_{A} T_{F} n\right]$. Appelquist and Georgi and Zee calculated the corrections to the scaling of the $e^{+}-e^{-}$annihilation cross section $[66,67]$. Gaillard and Lee [68], and independently Altarelli and Maiani [69], calculated the enhancement of the $\Delta I=\frac{1}{2}$ nonleptonic decay matrix elements. The analysis of scaling violations for deep-inelastic scattering continued [70], and the application of asymptotic freedom, what is now called perturbative $Q C D$, was extended to many new processes. The experimental situation developed slowly, and initially looked rather bad. I remember in the spring of 1974 attending a meeting in Trieste. There I met Burt Richter who was gloating over the fact that $R=\sigma_{e^{+} e^{-} \rightarrow \text { hadrons }} / \sigma_{e^{+} e^{-} \rightarrow \mu^{+} \mu^{-}}$was increasing with energy, instead of approaching the expected constant value. This was the most firm of all the scaling predictions. $R$ must approach a constant in any scaling theory. In most theories however one cannot predict the value of the constant. However, in an asymptotically free theory the constant is predicted to equal the sum of the squares of the charges of the constituents. Therefore if there were only the three observed quarks, one would expect that $R \rightarrow 3\left[\left(\frac{1}{3}\right)^{2}+\left(\frac{1}{3}\right)^{2}+\left(\frac{2}{3}\right)^{2}\right]=$ 2 . However Richter reported that $R$ was increasing, passing through 2 , with no sign of flattening out. Now many of us knew that charmed particles had to exist. Not only were they required, indeed invented, for the GIM mechanism to work, but as Claude Bouchiat, John Illiopoulos and Maini [71] and Roman Jackiw and I [72] showed, if the charmed quark were absent the electro-weak theory would be anomalous and non-renormalizable. Gaillard, Lee and Jonathan Rosner had written an important and insightful paper on the phenomenology of charm [73]. Thus, many of us thought that since $R$ was increasing probably charm was being produced. In 1974 the charmed mesons, much narrower than anyone imagined ${ }^{9}$ were discovered, looking very much like positronium-Coulomb bound states of quarks. This clinched the matter for many of the remaining skeptics. The rest were probably convinced once experiments at higher energy began to see quark and gluon jets. The precision tests of the theory, the logarithmic deviations from scal-

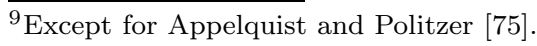


ing, took quite a while to observe. I remember very well a remark made to me by a senior colleague, in April of 1973 when I was very high, right after the discovery of asymptotic freedom. He remarked that it was unfortunate that our new predictions regarding deep-inelastic scattering were logarithmic effects, since it was unlikely that we would see them verified, even if true, in our lifetime. This was an exaggeration, but the tests did take a long time to appear. Confirmation only started to trickle in in $1975-78$; and then at a slow pace. By now the predictions are indeed verified, as we have heard at this meeting, in some cases to better than a percent. Nowadays, when you listen to experimentalists talk about their results they point to their lego plots and say, "Here we see a quark, here a gluon." Believing is seeing, seeing is believing. We now believe in the physical reality of quarks and gluons; we now believe in asymptotic simplicity of their interactions at high energies so we can see quarks and gluons. The way in which we see quarks and gluons, indirectly through the effects they have on our measuring instruments, is not much different from the way we see electrons. Even the objection that quarks and gluons can not be real particles, since they can never be isolated, has largely been dissipated. If we were to heat the world to a temperature of a few hundred $\mathrm{MeV}$, hadrons would melt into a plasma of liberated quarks and gluons.

\section{OTHER IMPLICATIONS OF ASYMPTOTIC FREEDOM.}

\subsection{Consistency of quantum field theory}

Traditionally, fundamental theories of nature have had a tendency to break down at short distances. This often signals the appearance of new physics that is discovered once one has experimental instruments of high enough resolution (energy) to explore the higher energy regime. Before asymptotic freedom it was expected that any quantum field theory would fail at sufficiently high energy, where the flaws of the renormalization procedure would appear. To deal with this, one would have to invoke some kind of fundamental length. In an asymptotically free theory this is not necessarily the case-the decrease of the effective coupling for large energy means that no new physics need arise at short distances. There are no infinities at all, the bare coupling is finite-indeed it vanishes. The only divergences that arise are an illusion that appears when one tries to compare, in perturbation theory, the finite effective coupling at finite distances with the vanishing effective coupling at infinitely short distances. Thus the discovery of asymptotic freedom greatly reassured one of the consistency of fourdimensional quantum field theory. One can trust renormalization theory for an asymptotically free theory, independent of the fact that perturbation theory is only an asymptotic expansion, since it gets better and better in the regime of short distances. We are very close to having a rigorous mathematical proof of the existence of asymptotically free gauge theories in four dimensions-at least when placed into a finite box to tame the infrared dynamics that produces confinement. As far as we know, QCD by itself is a totally consistent theory at all energies. Moreover, aside from the quark masses it has no arbitrary, adjustable parameters. ${ }^{10}$ Indeed, were it not for the electroweak interactions and gravity, we might be satisfied with QCD as it stands.

\subsection{Unification}

Almost immediately after the discovery of asymptotic freedom and the proposal of the nonAbelian gauge theories of the strong interactions, the first attempts were made to unify all the interactions. This was natural, given that one was using very similar theories to describe all the known interactions. Furthermore, the apparently insurmountable barrier to unification-namely the large difference in the strength of the strong interactions and the electro-weak interactions -was seen to be a low energy phenomenon. Since the strong interactions decrease in strength with increasing energy these forces could have a common origin at very high energy. Indeed in the fall of 1974 Georgi and Glashow proposed a unified theory, based on the gauge group $S U(5)$, which remarkably contained the gauge groups of the standard model as well as the quark and lepton multiplets

\footnotetext{
$\overline{{ }^{10}}$ This is one of the reasons it is so hard to solve.
} 
in an alluringly simple fashion [76] ${ }^{11}$ Georgi, Helen Quinn and Weinberg [78] showed that the couplings run in such a way as to merge somewhere around $10^{14}$ to $10^{16} \mathrm{GeV}$. This theory had the great advantage of being tight enough to make sufficiently precise predictions (proton decay and the Weinberg angle). It was a great stimulus for modern cosmology, since it implied that one could extrapolate the standard model to enormously high energies that corresponded to very early times in the history of the universe. Although the $S U(5)$ theory has been invalidated by experiment, at least in its simplest form, the basic idea that the next fundamental threshold of unification is set by the scale where the strong and electro-weak couplings become equal in strength remains at the heart of most attempts at unification.

\section{REFERENCES}

1. Landau, L.D., Fundamental Problems, in Pauli Memorial Volume, pg. 245, (Interscience, New York, 1960).

2. Feynman, R., in The Quantum Theory of Fields - The $12^{\text {th }}$ Solvay Conference, 1961 (Interscience, New York).

3. Landau, L.D. and I.Pomeranchuk, Dokl. Akad. Nauk SSSR 102, 489 (1955).

4. Landau, L.D., in Niels Bohr and the Development of Physics, (ed. W.Pauli), p. 52. (Pergamon Press, London, 1955).

5. Chew, G. in S-Matrix Theory, (W.A. Benjamin Inc, 1963).

6. Chew, G. in The Quantum Theory of Fields: The 12th Solvay Conference, 1961 (Interscience, New York).

7. Goldberger, M. in The Quantum Theory of Fields: The 12th Solvay Conference, 1961 (Interscience, New York).

8. Low, F., in Proceedings of the International Conference on High Energy Physics 1966, pg. 249 (1966).

9. Low, F., Suppl. al Nuovo Cimento Vol. IV,2,379 (1964).

10. Gell-Mann, M., Current Algebra: Quarks and

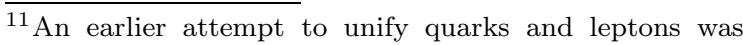
made by J. Pati and Salam [77].
}

What Else?, in Proceedings of the XVI Int'l Conference on High Energy Physics, Vol. 4, 135 (1972).

11. Gell-Mann, M. and Neeman, Y. The Eightfold Way (W.A. Benjamin Inc.) (1964).

12. Gell-Mann, M., The Symmetry Group of Vector and Axial Vector Currents, Physics, 1, 63 (1964).

13. Gell-Mann, M., A Schematic Model of Baryons and Mesons, Phys. Lett. 8, 214 (1964); Zweig, G., CERN Report No. TH401, 4R12 (1964) (unpublished).

14. Nambu,Y., Dynamical Symmetries and Fundamental Fields, Proceedings of the Second Coral Gables Conference on Symmetry Principles at High Energy , 133 (1965); A Systematics of Hadrons in Subnuclear OPhysics, in Preludes in Theoretical Physics, (1965)

15. Han, M.Y. and Nambu,Y., Three-Triplet Model with Double SU(3) Symmetry, Phys. Rev. 139B, 1006 (1965).

16. Greenberg, O.W., Spin and Unitary-Spin Independence in a Paraquark Model of Baryons and Mesons, Phys. Rev. Lett. 13, 598 (1964).

17. Dashen, R. and Gell-Mann, M., Representations of Local Current Algebra at Infinite Momentum, Phys. Rev. Lett. 17, 340(1966).

18. Adler, S., Sum Rules for the Axial Vector Coupling Constant Renormalization in $\beta$ Decay, Phys. Rev. 140 B, 736 (1965); Weisberger,W.I. Unsubtracted Dispersion Relations and the Renormalization of the Weak Axial-Vector Coupling Constants, Phys. Rev. 143 B, 1302 (1966).

19. Bjorken, J.D. Applications of the Chiral U(6) $\times U(6)$ Algebra of Current Densities, Phys. Rev. 148 B, 1467 (1966).

20. Bjorken, J., Current Algebra at Small Distances, Varenna School Lectures, Course XLI, Varenna, Italy (1967).

21. Bjorken, J., Inequality for Backward Electron and Muon-Nucleon Scattering at High Momentum Transfer, Phys. Rev. 163, 1767 (1967).

22. Sugawara, H., A Field Theory of Currents, Phys. Rev. 170 , 1659 (1968).

23. Callan, C.G. and Gross, D. J., Crucial Test of a Theory of Currents Phys. Rev. Lett. 21, 
311 (1968).

24. Bjorken, J., Asymptotic Sum Rules at Infinite Momentum, Phys. Rev. 179, 1547 (1969).

25. Bloom, E.D. et al., High-Energy Inelastic $e-p$ Scattering at $6^{\circ}$ and $10^{\circ}$, Phys. Rev. Lett. 23, 930 (1969).

26. Callan, C. G. and Gross, D. J., High-Energy Electroproduction and the Constitution of the Electric Current, Phys. Rev. Lett. 22, 156 (1968).

27. Gross, D.J. and Llewelyn-Smith,C., High Energy Neutrino-Nucleon Scattering, Current Algebra and Partons, Nucl. Phys. B14, 337 (1969).

28. Feynman, R. P., Very High-Energy Collisions of Hadrons, Phys. Rev. Lett. 23, 1415 (1969).

29. Drell, S.D. and Yan, T.M., Partons and Their Applications at High Energies, Ann. Phys.(NY) 66, 578 (1971).

30. Gross, D.J. and Wess, J., Scale Invariance, Conformal Invariance and the High Energy Behavior of Scattering Amplitudes, Phys. Rev. D2, 753 (1970).

31. Veneziano, G., Construction of a CrossingSymmetric Regge-Behaved Amplitude for Linearly Rising Trajectories, Nuovo Cimento, 57A, 190 (1968).

32. 't Hooft, G., Renormalizable Lagrangians for Massive Yang-Mills Fields, Nucl. Phys. 35, 167 (1967).

33. Gell-Mann, M. and Low, F., Quantum Electrodynamics at Small Distances, Phys. Rev. 95, 1300 (1954).

34. Stueckelberg, E. and Petermann, A., La Normalisation des Constantes dans la Theorie des Quanta, Helv. Phys. Acta 26, 499 (1953).

35. N. N. Bogoliubov and D. V. Shirkov, Introduction to the Theory of Quantized Fields, Interscience, New York, (1959).

36. Callan, C.G., Broken Scale Invariance in Scalar Field Theory,Phys. Rev D 2,1541 (1970); Symanzik, K., Small Distance Behavior in Field Theory and Power Counting, Comm. Math. Phys. 18, 227 (1970).

37. Wilson, K., Renormalization Group and Strong Interactions, Phys. Rev. D3, 1818 (1971).

38. Jackiw, R. Van Royen, R. and West, G., Mea- suring Light-Cone Singularities, Phys. Rev. D2, 2473 (1970); Frishman, Y., Operator Products at Almost Light Like Distances, Ann. Phys. 66, 373 (1971); Leutwyler, H. and Stern, J., Singularities of Current Commutators on the Light Cone, Nucl. Phys. B20,77 (1970); Gross, D. J., Light Cone Structure of Current Commutators in the Gluon-Quark Model, Phys. Rev. D4, 1059 (1971).

39. Callan, C.G., Broken Scale Invariance and Asymptotic Behavior, Phys. Rev D 5,3202 (1972); Symanzik, K., Small-DistanceBehavior Analysis and Wilson Expansions, Comm. Math. Phys. 23, 49 (1971); Christ, N., Hasslacher, B. and Mueller, A., Light-Cone Behavior of Perturbation Theory, Phys. Rev D6,3543 (1972).

40. Wilson, K. and Kogut, J., The Renormalization Group and the $\epsilon$ expansion. Phys. Rep. 31, 792 (1973).

41. Parisi, G., Deep-Inelastic Scattering in a Field Theory with Computable Large-Momenta Behavior, Lett. al Nuovo Cimento 7, 84 (1973).

42. Callan, C. G.and Gross, D. J., Bjorken Scaling in Quantum Field Theory, Phys. Rev. D8, 4383 (1973).

43. Coleman, S. and Gross, D. J., Price of Asymptotic Freedom, Phys. Rev. Lett. 31, 851 (1973).

44. Zee, A., Study of the Renormalization Group for Small Coupling Constants, Phys. Rev. D7, 3630 (1973).

45. Gross, D. J. and Wilczek, F., Ultraviolet Behavior of Non-Abelian Gauge Theories, Phys. Rev. Lett. 30, 1343 (1973).

46. Politzer, H.D., Reliable Perturbative Results for Strong Interactions?, Phys. Rev. Lett. 30, 1346 (1973).

47. Nielsen, N.K., Asymptotic Freedom as a Spin Effect, Am. J. Phys. 49, 1171 (1981).

48. Bardeen, W.A., Fritzsch, H. and Gell-Mann, M., Light-Cone Current Algebra, $\pi^{0}$ Decay, and $e^{+} e^{-}$Annihilation, in Scale and Conformal Symmetry in Hadron Physics, ed. R. Gatto (Wiley \& Sons, 1973).

49. Weinberg, S., Non-Abelian Gauge Theories of the Strong Interactions, Phys. Rev. Lett. 31, 
494 (1973).

50. Fritzsch, H., Gell-Mann, M. and Leutwyler, H., Advantages of the Color Octet Gluon Picture, Physics Lett. 47B, 368 (1973).

51. Fritzsch, H. and Gell-Mann, M., Current Algebra: Quarks and What Else?, in Proceedings of the XVI International Conference on High Energy Physics, Vol. 2, 164 (1972).

52. Belavin, A., Polyakov, A., Schwartz, A. and Tyupkin, Yu., Psuedoparticle Solutions of the Yang-Mills Equations, Phys. Lett. 59B, 85 (1975).

53. 't Hooft, G., Computation of the Quantum Effects due to a Four-Dimensional Psuedoparticle, Phys. Rev. D14, 3432 (1976).

54. Callan, C., Dashen, R. and Gross, D, The Structure of the Vacuum, Phys. Lett. (1976); Jackiw, R. and Rebbi, C. Vacuum Periodicity in a Yang-Mills Quantum Theory, Phys. Rev. Lett. 37, 172 (1976).

55. Gross, D. J. and Wilczek, F., Asymptotically Free Gauge Theories.I, Phys. Rev. D8, 3633 (1973).

56. Gross, D. J. and Wilczek, F., Asymptotically Free Gauge Theories.II, Phys. Rev. D9, 980 (1974).

57. Lipkin, H. Triality, Exotics and the Dynamical Basis of the Quark Model, Phys. Lett. Vol. 45B, 267 (1973).

58. Marciano, B. and Pagels, H., Quantum Chromodynamics, Phys. Rep. C 36 , 137 (1978).

59. Wilson, K., Confinement of Quarks, Phys. Rev. D10, 2445 (1974).

60. Creuz, M., Confinement and the Critical Dimensionality of Space-Time, Phys. Rev. Lett. 43, 553 (1968).

61. Gross, D. J. and Neveu, A., Dynamical Symmetry Breaking in an Asymptotically Free Theory, Phys. Rev. D10, 3235 (1974).

62. t' Hooft, G., A Planar Diagram Theory for Strong Interactions, Nucl. Phys. B72, 461 (1974).

63. A. Casher, J. Kogut and L. Susskind, Vacuum Polarization and the Quark-Parton puzzle, Phys. Rev. Lett. 31, 792 (1973); Vacuum Polarization and the Absence of Free Quarks, Phys. Rev. D 10, 732 (1974).

64. Caswell, W., Asymptotic Behavior of Non-
Abelian Gauge Theories to Two-Loop Order, Phys. Rev. Lett. 33, 244 (1974).

65. Jones, D. Two-Loop Diagrams in Yang-Mills Theory, Nucl. Phys. B75, 531, (1974).

66. Appelquist, T. and Georgi, H., $e^{+} e^{-}$Annihilation in Gauge Theories of Strong Interactions, Phys. Rev. D8, 4000 (1973).

67. Zee, A., Electron-Positron Annihilation in Stagnant Field Theories, Phys. Rev. D8, 4038, (1973).

68. Gaillard, M.K. and Lee, B.W. $\Delta I=\frac{1}{2}$ Rule for Non-Leptonic Decays in Asymptotically Free Field Theories, Phys. Rev. Lett 33, 108 (1974).

69. Altarelli, G. and Maiani, L., Octet Enhancement of Non-Leptonic Weak Interactions in Asymptotically Free Gauge Theories, Phys. Lett. 52B, 351 (1974).

70. Gross, D. J., How to Test Scaling in Asymptotically Free Theories, Phys. Rev. Lett. 32, 1071 (1974).

71. Bouchiat,C., Iliopoulpos, J. and Meyer, Ph., An Anomaly Free Version of Weinberg's Model, Phys. Lett. 38B, 519 (1972).

72. Gross, D.J. and Jackiw, R., The Effect of Anomalies on Quasi-Renormalizable Theories, Phys. Rev. D6, 477 (1972).

73. Gaillard, M.K., Lee, B.W. and Rosner, J.L., Search for Charm, Rev. Mod. Phys. 47, 277 (1975).

74. S. Adler, Phys. Rev. 177, 2426 (1969); J. Bell and R. Jackiw, Nuovo Cimento 60A, 47 (1969).

75. Appelquist, T. and Politzer, H. D., Heavy Quarks and $e^{+} e^{-}$Annihilation, Phys. Rev. Lett. 34, 43 (1975).

76. Georgi, H. and Glashow, S., Unity of All Elementary Particle Forces, Phys. Rev. Lett. 32 , 438 (1974).

77. Pati, J.C. and Salam, A., Unified LeptonHadron Symmetry and a Gauge Theory of the Basic Interactions, Phys. Rev. D 8 , 1240 (1973).

78. Georgi, H., Quinn, H.R. and Weinberg, S., Hierarchy of Interactions in Unified Gauge Theories, Phys. Rev. Lett. 33, 451 (1974). 